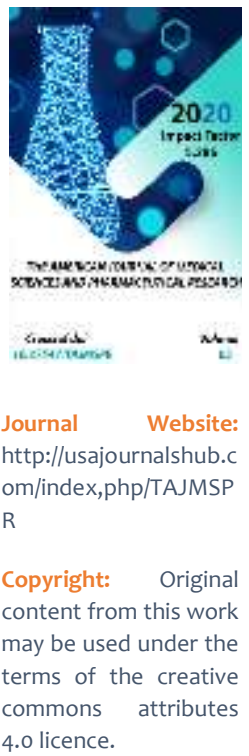

\title{
PLGF/ SFLT-1 Concentrations As Early Predictors Preeclampsia In Pregnancy During Pandemic Covid-19
}

\author{
Makhmudova Sevara Erkinovna \\ Samarkand State Medical Institute, Uzbekistan \\ Negmadjanov Baxodur Boltaevich \\ Samarkand State Medical Institute, Uzbekistan
}

\section{ABSTRACT}

The imbalance in placenta- originated angiogenic factors has been found to play an important role in the pathogenesis of preeclampsia in recent years. Soluble fms- like tyrosine kinase 1 (sFlt-1) and placental growth factor (PIGF) are the most studied proteins. sFlt-1, a splice variant of the vascular endothelial growth factor (VEGF) receptor Flt-1, is a circulating antiangiogenic protein that inhibits proangiogenic factors - VEGF and PIGF signalling in the vasculature. High levels of circulating sFlt-1 and low levels of PIGF were observed in women with established preeclampsia and even before the onset of clinical symptoms $[1,6,9,11]$.

\section{KEYWORDS}

PIGF/ SFLT-1 concentrations, placenta, preeclampsia, pregnancy, proangiogenic factors.

\section{INTRODUCTION}

Numerous studies focused on the pathological effects of excess sFlt-1 on endothelial function. It was observed that overexpression of sFlt-1 produced a preeclampsia- like syndrome of hypertension, proteinuria and glomerular endotheliosis in experimental animals. 5 sFlt- 1 is largely produced by syncytiotrophoblast and secreted into maternal circulation.6 Placental hypoxia may be one of the main triggers of inducing abundant sFlt-1 expression and 
leading to hypertensive complications. However, this hypothesis may not totally explain why women with elevated blood pressure (BP) before pregnancy have a substantially higher risk of preeclampsia $[4,7,11]$

Preeclampsia is known to be one of the very serious and fairly common (5-8\%) complications of pregnancy. Preeclampsia is characterized by an increase in blood pressure (more than $140 / 90 \mathrm{mmHg}$ ) and an increase in protein concentration (more than $100 \mathrm{mg} / \mathrm{dL}$ in random urine samples collected at $4 \mathrm{~h}$ intervals) [4]. The etiopathogenesis of preeclampsia has not yet been definitively clarified. There are about 30 theories of the development of this pathology, but in our view one deserves special attention $(3,5,8,10)$. It has been established that the placenta formation process begins with the movement of large numbers of trophoblasts against the blood flow in the small spiral arteries of the uterus. These cells stimulate the synthesis of cytokines, growth factors and adhesion molecules, resulting in the expansion of spiral arteries and the formation of larger-diameter blood vessels capable of providing the developing fetus with oxygen and the necessary nutrients $[6,12]$. This process begins at the end of the first trimester and ends in 18-20 weeks [1, 2, 11]. If the initial flow of trophoblasts is abnormally small, the spiral arteries are incompletely reconstructed, which may cause inadequate blood supply to the developing fetus and the development of pre-eclampsia. In order to compensate for the lack of blood supply to the fetus, soluble factors in the placenta begin to be synthesized, which enter the maternal bloodstream. However, these factors lead to systemic endothelial dysfunction, hypertension and proteinuria development $[9,10,12]$
It has been shown that a decrease in PIGF concentration and an increase in sflt-1 concentration are recorded long before the clinical signs of pre-eclampsia occur and can be a screening test as early as the end of the first trimester of pregnancy. However, data on the concentration of these markers and their ratio in physiological pregnancy dynamics are only given in a few publications $[1,9,12]$. In connection with the above, the aim of this study was to develop reference limits for sflt- 1 and PIGF concentrations and ratios in the first, second and third trimesters of physiological pregnancy for early diagnosis of preeclampsia, and to demonstrate the informative value of these tests in moderate to severe preeclampsia.

\section{MATERIALS AND METHODS}

The concentrations of PIGF and sflt-1 in the serum of pregnant women were determined using the electrochemiluminescent diagnostic test systems elecsys PIGF and elecsys sflt-1 of Hoffmann La Roche. (Switzerland) on an automatic cobas e411 analyser. Surveys were carried out:

- In the first trimester - 78 patients with a period of physiological pregnancy of 11-13 weeks, who according to ultrasound data have a normally developing fetus and low risk of fetal pathology according to the results of prenatal diagnosis;

- In the second trimester - 124 patients with a period of physiological pregnancy of 1620 weeks, who have no pathology of fetal development.

- In the third trimester of pregnancy - 75 patients with a period of physiological pregnancy of 30-39 weeks who have no pathology of fetal development. Blood was received from the ulnar vein. Blood pressure levels in all patients during 
pregnancy did not exceed the normative range, and protein was not registered in the urine. All women gave birth to healthy infants with an Apgar score of 8-9 within 38-40 weeks. The early neonatal period in all children proceeded without complications.

The concentrations of sflt-1 do not differ significantly from those in the second trimester of pregnancy. The ratio of sflt-1/PIGF in the first trimester was 39.3 \pm 4.2 . The dynamics of PIGF and sflt-1 concentrations and their ratios in the second and third trimesters of pregnancy are presented in Tables 2 and 3. The concentrations of PIGF and sflt-1 in 16 weeks did not differ significantly from those in 17 and 18 weeks of pregnancy. The concentration of PIGF increased reliably in 19 weeks, while in 20 weeks it exceeded reliably in 19 weeks. The ratio of PIGF and sflt-1 concentrations also changed accordingly. In 16-18 weeks of pregnancy, the sflt-1/PIGF ratio averaged 13.1 \pm 2.6 , while in 19-20 weeks it was 6.9 \pm 2.1 . It should be noted that in the first trimester of pregnancy (11-13 weeks), the average value of this indicator was $39.3 \pm 4.2$. During the third trimester of pregnancy, the following features of PIGF and sflt-1 concentrations were observed. In 30-32 weeks, the PIGF concentration was more than twice as high as in 20 weeks of pregnancy, and the concentrations of sflt-1 did not differ significantly in these gestational periods. Accordingly, the sflt-1/PIGF ratio in 30-32 weeks was minimal and averaged $1.7 \pm 0.8$. Similar results were obtained by a group of researchers from the Department of Obstetrics and the Hospital of the University of Leipzig, Germany, who also used Hoffman La Roche diagnostic test systems. [12]. In 33-36 weeks of pregnancy, the PIGF concentration was 3 times lower than in 30-32 weeks, and the sflt-1 concentration doubled. The ratio of these indicators was 10 \pm 2.5 . In 37-40 weeks of pregnancy, there was a further decrease in PIGF concentration and an increase in sflt-1 concentration sflt-1. Their ratio was 17.6 \pm 2.4 .

Thus, as a result of the work carried out, data were obtained on the content of PIGF and sflt1 and the values of their ratio in pregnancy terms from 11 to 13 weeks, from 16 to 20 weeks and from 30 to 40 weeks. The gestational dates selected for the study were due to the fact that it is at this time that routine prenatal diagnosis is carried out in the first and second trimesters, as well as dynamic monitoring of the fetus in the third trimester of pregnancy. It seems that assessing the risk of pre-eclampsia development during these gestational periods can help reduce the frequency of complications and perinatal losses, since taking appropriate measures in a number of cases makes it possible to correct the developing pathological condition in a timely manner and resolve the issue of the timing of delivery. An analysis of the data obtained indicates that there are reliable differences between PIGF concentrations and sflt-1.

\section{RESULT AND DISCUSSION}

PIGF in pregnant women with pre-eclampsia and corresponding reference rates already in the first trimester of pregnancy. Four out of six patients with severe pre-eclampsia had sflt1/PIGF ratio values more than 3 times higher than the corresponding standard. Patients with moderate pre-eclampsia who developed pregnancy dynamics had values slightly higher than the norm. Two patients with moderate pre-eclampsia were able to prolong the pregnancy to 36-38 weeks, while those with severe pre-eclampsia were able to give birth by Caesarean section much earlier (26-34 weeks) to save the life of the pregnant woman and fetus. A comparison of the results of the study of these patients in the first and third 
trimesters of pregnancy (samples in the third trimester were obtained prior to delivery) shows that the concentration of sflt-1 increased sharply in the dynamics of pregnancy (3-10 times), while the increase in PIGF concentration was less pronounced (1.5-4 times). In two patients, PIGF concentrations in the first trimester and on the day of delivery at 27 and 26 weeks, respectively, did not differ significantly. A sharp increase in sflt-1 concentrations and a slight increase in PIGF concentrations led to an increase in their ratio, which was 1,109.0 and 371.2. The live births of these women had a body weight of 450 and $500 \mathrm{~g}$ and a low Apgar rating. Unfortunately, the remaining 16 women with pre-eclampsia were unable to obtain data on sflt-1 and PIGF content in the first or second trimester of pregnancy. They were provisionally diagnosed with pre-eclampsia based on clinical findings. The timing of delivery was determined based on clinical indications and data on the state of the fetus. The following was established by comparing the data obtained on sflt-1 and PIGF concentrations in the blood of these women with the corresponding reference values. The concentration of sflt-1 was 3-10 times higher than the corresponding standard for the given pregnancy period. The PIGF concentration was only in 4 out of 16 cases that met the standard. In 12 cases, the PIGF concentration was reduced by a factor of 2-7. As a result of these changes, the sflt-1/PIGF ratio was 4-20 times higher than the reference indicator. It is known that the final diagnosis of preeclampsia cannot be made solely on the basis of blood pressure and protein levels in blood and urine $(4,10,13)$. Elevated blood pressure may be due to hypertension in pregnant women, and increased protein concentration may be due to kidney pathology (2). Since the etiopathogenesis of preeclampsia is due to the development of endothelial insufficiency and vascular disorders in the mother-placentafetus system, the choice of such angiogenesis indicators as sflt-1 and PIGF as screening tests is justified logically $[7,9,11]$. The results of the practical application of this approach to the diagnosis of the threat of pre-eclampsia indicate a high degree of informativeness in analysing data on the content of these markers and their ratio in the blood of pregnant women $[1,11-14]$. The increase in sflt-1 concentration appears to be disrupting the intracellular mechanism of regulation of PIGF synthesis, which in turn leads to the development of systemic endothelial insufficiency and, as a result, to a delay in fetal intrauterine development $[3,6]$. The results of this study clearly confirm this fact. Indeed, as early as the first trimester of pregnancy, it is possible to diagnose the imbalance in the synthesis of sflt1 and PIGF, which increases the value of the ratio. In the dynamics of pregnancy, it is usually not possible to achieve normalization of the vascular system without appropriate measures to correct this pathology, which leads to a delay in fetal development, the development of kidney pathology in a pregnant woman and an increase in blood pressure. In turn, the development of clinical signs of pre-eclampsia and the degree to which they are pronounced lead to the need to immediately address the question of whether to maintain the pregnancy in question or to take measures to prevent preeclampsia [2]. In the third trimester of pregnancy, the definition of the indicators studied and the analysis of data on their correlation may be the final stage of the diagnosis $[1,11,13]$.

\section{CONCLUSIONS}

1. The sflt-1 and PIGF concentrations and their ratios are highly informative indicators of pre-eclampsia. 
2. Determination of the concentration of these markers and the calculation of their ratio should be carried out in the first and second trimesters of pregnancy as part of a screening programme to diagnose fetal pathology. This will help to resolve the issue of the advisability of maintaining this pregnancy and the tactics of preventing pre-eclampsia.

3. The determination of preeclampsia markers in the third trimester of pregnancy can serve as a basis for the final diagnosis and decision on the timing of delivery in order to preserve the life of the woman and the fetus.

\section{REFERENCES}

1. Bergmann A, Ahmad S, Cudmore $M$, Gruber AD, Wittschen $P$, Lindenmaier $W$, et al. Reduction of circulating soluble Flt-1 alleviates preeclampsialike symptoms in a mouse model. $J$ Cell Mol Med. 2010;14:1857-67.

2. Chan IH, Leung TF, Tang NL, Li CY, Sung YM, Wong GW, et al. Gene-gene interactions for asthma and plasma total IgE concentration in Chinese children. J Allergy Clin Immunol. 2006;117:127-33.

3. David-Ona D, De Castro DM, Baltazar AC. The distribution of hypertension in the Philippine general hospital after 4 decades (a comparative study). Acta Med Philipp. 2013;47(3):49-52.

4. Dickson ME, Sigmund CD. Genetic basis of hypertension: revisiting angiotensinogen. Hypertension. 2006;48:14-20.

5. $\quad$ Khan KS, Wojdyla D, Say L, Gulmezoglu AM, Van Look PF. WHO analysis of causes of maternal death: a systematic review. Lancet. 2006;367:1066-74. 3.

6. Mikhail MS, Anyaegbunain A, Garfinkel D, Palan PR, Basu J, Roinney SL. Preeclanmpsia and antioxidant nutrients: Decreased plasmia levels of reduced ascorbic acid, alphatocopherol. and beta-carotene in women with preeclanipsia. Am J Obstct Gynaecol 1994:171:150;

7. Okby R, Harlev A, Sacks KN, Sergienko $\mathrm{R}$, Sheiner E.Preeclampsia acts differently in in vitro fertilization versus spontaneous twins.Arch Gynecol Obstet. 2018 Jan 4. doi: 10.1007/s00404-017-4635-y.

8. Powe C.E., Levine R.J., Karumanchi, S.A. Preeclampsia, a disease of the maternal endothelium. The role of antiangiogenic factors and implications for later cardiovascular disease. Circulation 2011; 123: 2856-2869.

9. Rahimi Z, Zangeneh $M$, Rezaeyan $A$, Shakiba E, Rahimi Z.MMP-8 C-799T and MMP-8 $\mathrm{C}+17 \mathrm{G}$ polymorphisms in mild and severe preeclampsia: Association between MMP-8 C-799T with susceptibility to severe preeclampsia.Clin Exp Hypertens. 2018;40(2):175-178. doi: 10.1080/10641963.2017.1346115. Epub 2017 Jul 26.

10. Rao H, Bai Y, Zhang F, Li Q, Zhuang B, Luo $X$, Qi H.The role of SATB1 in HTR8/SVneo cells and pathological mechanism of preeclampsia.J Matern Fetal Neonatal Med. 2018 Jan 7:1-151.

11. Schiff E, Friedm-an SA, Stamapfer $M$, Kao L, Barre tt PH, Sibai BM. Dietary consumimptiori and plasmla concentration s of vitarlin $E$ in pregnancies complicated by 
preeclanilpsia. Am J Obstet Gynecol 199)6; $175: 10 f 24-8$

12. Shin $\mathrm{YY}$, Jeong JS, Park MN, Lee JE, An SM, Cho WS, Kim SC, An BS, Lee KS.Regulation of steroid hormones in the placenta and serum of women with preeclampsia.Mol Med Rep. 2018 Feb;17(2):2681-2688. doi: 10.3892/mmr.2017.8165. Epub 2017 Nov 27. 